\title{
Theaflavins suppress tumor growth and metastasis via the blockage of the STAT3 pathway in hepatocellular carcinoma
}

This article was published in the following Dove Press journal:

OncoTargets and Therapy

14 July 2016

Number of times this article has been viewed

\author{
Jianping Shaol,* \\ Qingyan Meng',* \\ Yongyuan $\mathrm{Li}^{\prime}$
}

'Department of General Surgery, ${ }^{2}$ Outpatient Department, The Fifth Central Hospital of Tianjin, Tianjin, People's Republic of China

*These authors contributed equally to this work
Correspondence: Jianping Shao Department of General Surgery, The Fifth Central Hospital of Tianjin, No 4 I Tangguzhejiang Road, Binhai New District, 300450 Tianjin,

People's Republic of China

Tel/fax +862265665302

Email shaojianping@163.com

\begin{abstract}
Theaflavins, the major black tea polyphenols, have been reported to exhibit promising antitumor activities in several human cancers. However, the role of theaflavins in hepatocellular carcinoma (HCC) is still unknown. In this study, we found that theaflavins could significantly inhibit proliferation, migration, and invasion, and induce apoptosis in HCC cells in vitro. Furthermore, we found that theaflavins inhibited the growth and metastasis of HCC in an orthotopic model and a lung metastasis model. Immunohistochemical analyses and terminal deoxynucleotidyl transferase dUTP nick end-labeling assays showed that theaflavins could suppress proliferation and induce apoptosis in vivo. Theaflavins also suppressed constitutive and inducible signal transducer and activator of transcription 3 (STAT3) phosphorylation. The downstream proteins regulated by STAT3, including the antiapoptotic proteins (Bcl-2 and Survivin) and the invasion-related proteins (MMP-2, MMP-9), were also downregulated after theaflavins treatment. Theaflavins induced apoptosis by activating the caspase pathway. Together, our results suggest that theaflavins suppress the growth and metastasis of human HCC through the blockage of the STAT3 pathway, and thus may act as potential therapeutic agents for HCC.
\end{abstract}

Keywords: theaflavins, tumor growth, tumor metastasis, STAT3, hepatocellular carcinoma

\section{Introduction}

Hepatocellular carcinoma (HCC) is the sixth most common malignancy and the third leading cause of cancer-related death worldwide. ${ }^{1}$ Although epidemiological studies have shown that chronic viral infections and hepatotoxic agents are the major risk factors, the molecular pathogenesis of HCC involves several oncogenes and tumor suppressor genes. ${ }^{2,3}$ Current treatment options, including surgical resection and conventional chemotherapy, are commonly associated with severe morbidity, side effects, and the development of drug resistance. ${ }^{4}$ Novel drugs are therefore needed for better prevention and treatment of HCC.

Natural compounds or their derivatives are still one of the major sources of antitumor drugs. Although the development of synthetic chemistry has led to the production of novel drugs, treatments for cancer cannot totally replace the potent biological activities and great structural diversity of natural products. ${ }^{5}$ Theaflavins, derived from black tea, are thought to possess antioxidant properties, the ability to scavenge or inhibit free radicals, and the ability to treat blood vessel and heart diseases. ${ }^{6-9}$ Recent studies have reported that theaflavins exhibit antitumor activities on different tumor cells, including breast carcinoma, prostate carcinoma, and melanoma. ${ }^{10-15}$ Theaflavins also exert their protective effect against dimethylnitrosamine-induced liver fibrosis, 
which is a risk factor for developing HCC. ${ }^{16}$ However, the exact role of theaflavins in HCC is still unclear.

The purpose of this study was therefore to investigate the effects of theaflavins on the proliferation, migration, and invasion of HCC in vitro and in vivo, as well as to characterize the associated mechanisms of these processes.

\section{Materials and methods}

\section{Cell lines, reagents, and antibodies}

HCCLM3, Huh-7, and LO2 cell lines were purchased from the Cell Resource Center of Shanghai Institute for Biological Sciences, Chinese Academy of Sciences (Shanghai, People's Republic of China). The cells used in this paper were approved by the Committee of the Fifth Central Hospital of Tianjin. All the cells were cultured in Dulbecco's Modified Eagle's Medium (Thermo Fisher Scientific, Waltham, MA, USA) supplemented with $10 \%$ fetal bovine serum. Theaflavins, interleukin-6 (IL-6), and benzyloxycarbonyl-valinyl-alaninyl-aspartyl fluoromethyl ketone were purchased from Sigma-Aldrich Co. (St Louis, MO, USA). NSC 74859 was obtained from Santa Cruz Biotechnology Inc. (Dallas, TX, USA). Primary antibodies against Bcl-2, Survivin, cleaved-caspase-3, cleaved-caspase-9, cleaved-poly (ADP-ribose) polymerase (PARP), pro-caspase-3, pro-PARP, p-ERK, ERK, p-AKT, AKT, p-STAT3, and STAT3 were purchased from Cell Signaling Technology (Danvers, MA, USA). Glyceraldehyde 3-phosphate dehydrogenase and secondary antibodies against mouse IgG-horseradish peroxidase (HRP) and rabbit IgG-HRP were obtained from Santa Cruz Biotechnology. Antibodies against Ki-67, MMP-9, and MMP-2 were obtained from Abcam (Cambridge, UK).

\section{Cell viability assay}

Cell viability was determined by a Cell Counting Kit-8 (CCK-8) assay (Dojindo Laboratories, Kumamoto, Japan). Briefly, cells were incubated in a 96-well plate at a density of $3 \times 10^{3}$ cells per well and treated with different concentrations of theaflavins $(0,10,20,40,60$, or $80 \mu \mathrm{g} / \mathrm{mL})$ for 24 or 48 hours. The supernatant of the cell culture was discarded, and a mixture of CCK-8 and a new cell culture was added to each well. After incubation at $37^{\circ} \mathrm{C}$ for 2 hours, the absorbance was measured using a microplate reader at $450 \mathrm{~nm}$.

\section{Cell cycle and apoptosis analysis}

The cell cycle analysis kit and annexin V-fluorescein isothiocyanate apoptosis kit were purchased from BD Biosciences (San Jose, CA, USA). For cell cycle analyses, cells were treated with theaflavins $(40 \mu \mathrm{g} / \mathrm{mL})$ for 48 hours, and then $1 \times 10^{6}$ cells were fixed in $70 \%$ ethanol at $-20^{\circ} \mathrm{C}$ overnight. On the next day, the fixed cells were stained according to the protocol of the manufacturer and analyzed by flow cytometry (Beckman Coulter FC500; Beckman Coulter, Brea, CA, USA). For apoptosis analyses, cells were harvested at a concentration of $1 \times 10^{6}$ cells $/ \mathrm{mL}$ after treatment with theaflavins $(40 \mu \mathrm{g} / \mathrm{mL})$ for 48 hours. The cells were then stained according to the protocol of the manufacturer and analyzed by flow cytometry using the Beckman Coulter FC500.

\section{Wound-healing assay}

HCC cells were seeded at $5-6 \times 10^{5}$ cells per well in a sixwell plate and allowed to grow to confluence. The cells were then washed once in medium, scratched with a $10 \mu \mathrm{L}$ pipette tip, and then incubated in growth medium with $20 \mu \mathrm{g} / \mathrm{mL}$ of theaflavins. Images were taken at 0 and 36 hours at $\times 10$ using a Nikon Eclipse TS100 microscope (Nikon, Tokyo, Japan).

\section{Cell invasion assay}

HCC cells were pretreated with $20 \mu \mathrm{g} / \mathrm{mL}$ of theaflavins for 24 hours. Then, invasion was measured using 24-well BioCoat cell culture inserts (BD Biosciences) with a polyethylene terephthalate membrane of $8 \mu \mathrm{m}$ porosity coated with a Matrige ${ }^{\circledR}$ basement membrane.

\section{Western blotting}

HCCLM3 and Huh- 7 cells were plated at a density of $3 \times 10^{5}$ cells/well in six-well plates. After incubation with different doses of theaflavins, the cells were washed twice with ice-cold phosphate-buffered saline (PBS) and treated with $200 \mu \mathrm{L}$ sample buffer on ice for 30 minutes. The cell lysate was centrifuged at $12,000 \mathrm{rpm}$ for 10 minutes at $4^{\circ} \mathrm{C}$. Protein lysates $(30 \mu \mathrm{L})$ were electrophoresed on a $12 \%$ or $10 \%$ sodium dodecyl sulfate gel. The proteins were then electrophoretically transferred to a polyvinylidene difluoride membrane, and the membrane was blocked with 5\% nonfat dry milk for 2 hours. The membrane was then incubated overnight at $4^{\circ} \mathrm{C}$ with primary antibodies (1:1,000-1:4,000 dilutions). Subsequently, the membrane was washed in PBS containing Tween 20 for 30 minutes, exposed to HRPconjugated secondary antibody (diluted to 1:2,000), and washed again in PBS containing Tween 20 for 30 minutes. Final detection was performed using an enhanced chemiluminescence solution (Pierce, Waltham, MA, USA).

\section{Immunohistochemical analysis}

Frozen $5 \mathrm{~mm}$ thick sections of tumor samples were prepared to determine the proliferation ability with anti-Ki-67 antibody (1:50 dilution). After blocking endogenous peroxidase activity, 
the sections were incubated overnight with the primary antibody at $4{ }^{\circ} \mathrm{C}$. Detection was done using the Polink-2 Plus IHC Detection System (Beijing Zhongshan Biotechnology, Beijing, People's Republic of China) according to the manufacturer's instructions. Positively stained cells were counted in ten randomly selected fields under $\times 400$ high-power magnification. A proliferative index, expressed in percentage of cells, was calculated using the formula: number of Ki-67-positive cells/total cell count.

\section{Terminal deoxynucleotidyl transferase dUTP nick end-labeling assay}

Apoptosis was detected using a terminal deoxynucleotidyl transferase dUTP nick end-labeling enzyme kit (Hoffman-La Roche Ltd., Basel, Switzerland) according to the manufacturer's instructions.

\section{Orthotopic animal model of HCC}

Male BALB/c athymic nude mice (4-6 weeks old) were obtained from the experimental animal center of Shanghai Institute for Biological Sciences and housed under standard conditions according to the institutional guidelines for animal care. All surgical procedures and care administered to the animals were approved by the institutional ethics committee. HCCLM3 cells $\left(5 \times 10^{5}\right)$ were subcutaneously injected into the backs of mice $(n=6)$ to establish tumors. Two weeks later, subcutaneous tumors were cut into pieces $\left(1 \mathrm{~mm}^{3}\right.$ each $)$ under aseptic conditions and then implanted into the left liver lobe of nude mice. Mice received daily $200 \mu \mathrm{L}$ intraperitoneal injections of either PBS (control) or theaflavins $(10 \mathrm{mg} / \mathrm{kg} / \mathrm{d})$. The dose and method of administration were based on our preliminary experiments. After 5 weeks of treatment, the mice were euthanized, and the tumor volume was calculated using the following equation: tumor volume $=$ length $\times(\text { width })^{2} \times \pi / 6$. The tumor tissue was then prepared for further analyses.

\section{In vivo experimental metastasis assay}

All of the animal experiments were approved by the Institutional Animal Care and Use Committee of the Fifth Central Hospital of Tianjin, and all mice were housed under standard conditions according to the guidelines of the Institutional Animal Care and Use Committee. To establish the lung metastasis model, eight mice in each group were injected with HCCLM3 cells $\left(2.5 \times 10^{6}\right)$. Mice were treated with theaflavins $(10 \mathrm{mg} / \mathrm{kg} / \mathrm{d})$ for 6 weeks. PBS was used as a control. At the end of the treatment, mice were euthanized, and the tumor nodules formed on the lungs were counted. Lungs were excised to perform further experiments.

\section{Statistical analyses}

All data are expressed as the mean \pm standard deviation. Comparisons among multiple groups were made with a one-way analysis of variance followed by Dunnett's $t$-test. A $P$-value $<0.05$ was considered significant.

\section{Results}

\section{Theaflavins inhibit proliferation and induce apoptosis in HCC cells}

We first performed a CCK-8 assay to detect the antiproliferative effect of theaflavins on HCC cells. HCC cells were cultured for 24 hours in different concentrations $(0,10,20,40,60$, or $80 \mu \mathrm{g} / \mathrm{mL}$ ) of theaflavins, and then the cell viability was measured. Theaflavins significantly inhibited the viability of HCC cell lines in a dose-dependent manner (Figure 1A). Extending drug exposure to 48 hours resulted in additional cytotoxicity, indicating that theaflavins also suppressed viability of HCC cells in a time-dependent manner (Figure $1 \mathrm{~B}$ ). The $\mathrm{IC}_{50}$ values (half-maximal inhibitory concentration) of theaflavins after exposure to the drug for 48 hours were 44.01 and $41.58 \mu \mathrm{g} / \mathrm{mL}$ for HCCLM3 and Huh-7 cells, respectively. However, this suppressive effect was not observed in the normal human liver cell line, LO2, at these treatment doses for both 24 and 48 hours (Figure 1A and B). To determine whether HCC cell death induced by theaflavins involved apoptosis, flow cytometric analyses with annexin V-propidium iodide staining were performed. Theaflavins induced apoptosis in HCCLM3 and Huh-7 cells after a 48-hour treatment (Figure 1C and D). Similar results were observed in HepG2 cells with differential biological and genetic characteristics (Figure S1A). Then, flow cytometric analyses were performed to evaluate the effect of theaflavins on cell cycle progression. There was no apparent change in the distribution of the HCC cell cycle after treatment with theaflavins (Figure 1E). Together, these data show that theaflavins inhibit proliferation of HCC cells through the induction of apoptosis.

\section{Theaflavins induce cell apoptosis in a caspase-dependent manner}

Theaflavins significantly increased the expression of cleaved-caspase-9, cleaved-caspase-3, and cleaved-PARP in a dose-dependent manner, and treatment with theaflavins led to a dose-dependent decrease of pro-caspase- 3 and pro-PARP (Figure 2A). To explore the dependence of theaflavin-induced apoptosis on the caspase pathway, HCC cells were pretreated with a pan-caspase inhibitor, Z-VADFMK $(10 \mathrm{mmol} / \mathrm{L})$, before treatment with theaflavins. The pretreatment with Z-VAD-FMK partially reduced theaflavininduced apoptosis as determined by annexin V-propidium 
A

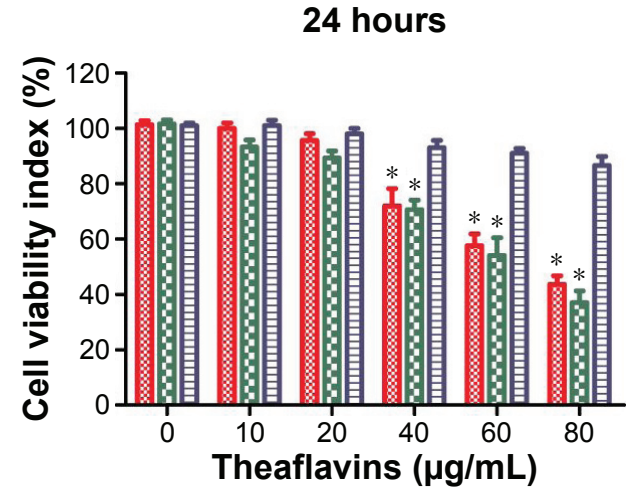

B

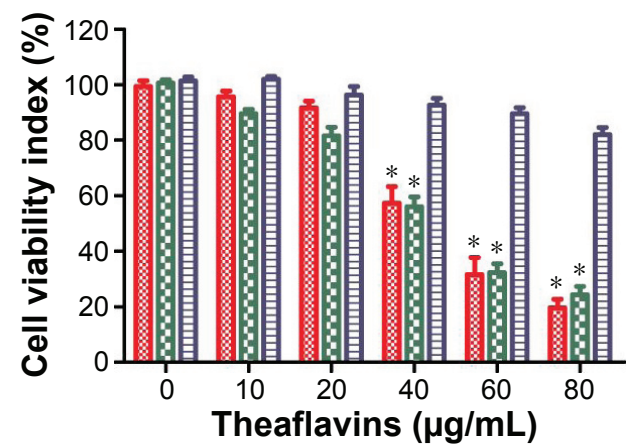

HCCLM3

C

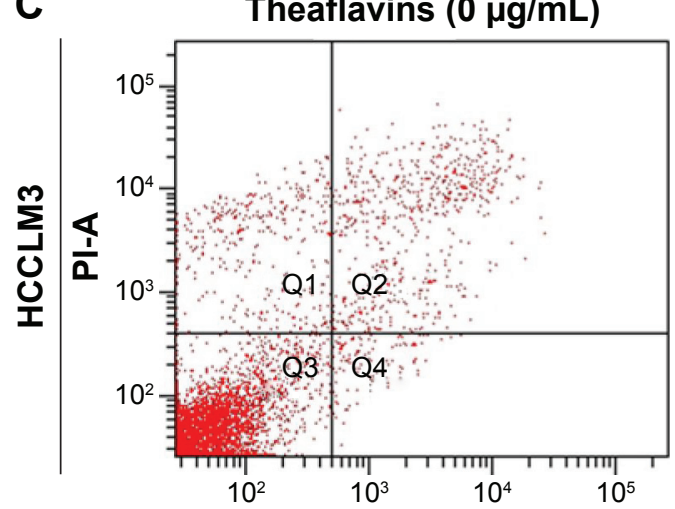

FITC-A

D

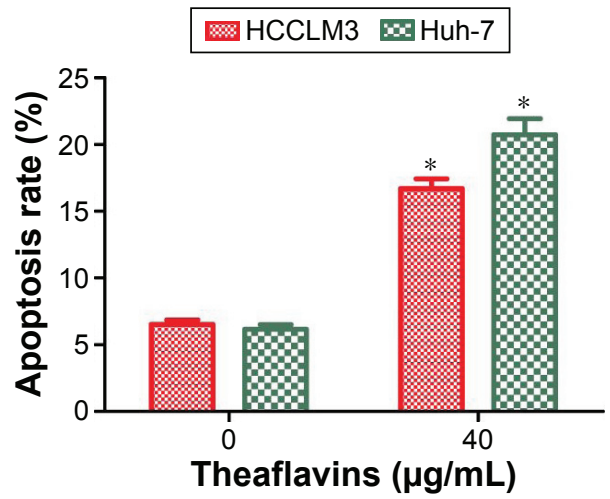

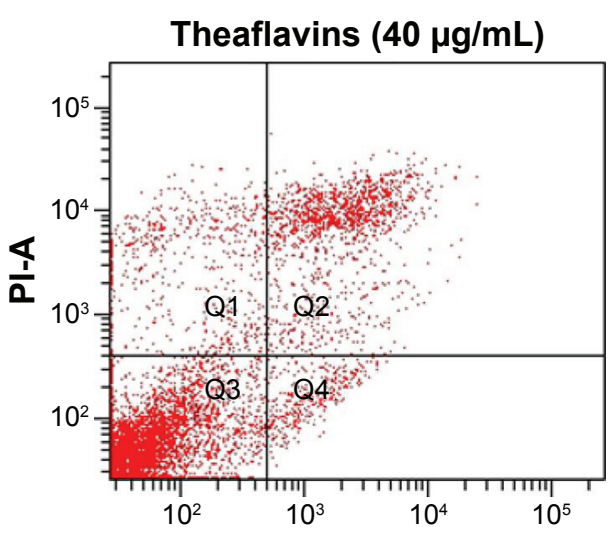

FITC-A

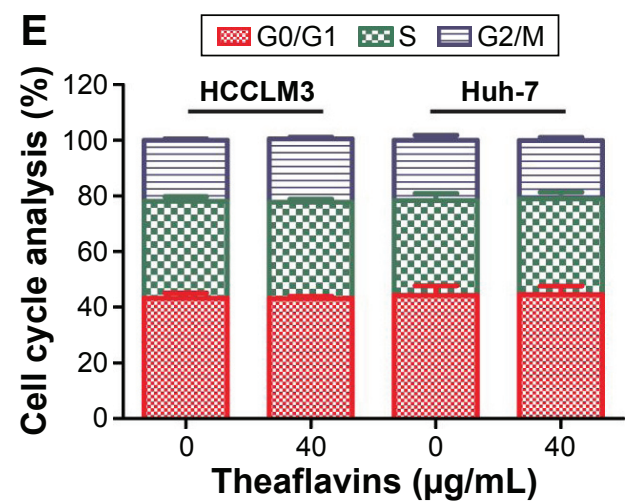

Figure I Theaflavins inhibit proliferation and induce apoptosis in $\mathrm{HCC}$ cells.

Notes: (A) HCC and LO2 cells were treated with 0, 10, 20, 40, 60, or $80 \mu \mathrm{g} / \mathrm{mL}$ theaflavins for 24 hours, and cell viability was measured using the CCK-8 assay. (B) HCC and LO2 cells were treated with $0,10,20,40,60$, or $80 \mu g / m L$ of theaflavins for 48 hours, and cell viability was measured using the CCK-8 assay. (C) A representative example of apoptosis of HCCLM3 cells treated with $40 \mu \mathrm{g} / \mathrm{mL}$ of theaflavins for 48 hours. (D) Flow cytometry results of annexin V-PI-stained HCC cells after exposure to $40 \mu \mathrm{g} / \mathrm{mL}$ of theaflavins for 48 hours. (E) Histograms of the cell cycle distribution of HCC cells treated with $40 \mu \mathrm{g} / \mathrm{mL}$ of theaflavins for 48 hours. Data are expressed as the mean \pm SD of three independent experiments. $* P<0.05$.

Abbreviations: HCC, hepatocellular carcinoma; CCK-8, Cell Counting Kit-8; SD, standard deviation; PI, propidium iodide; A, annexin; FITC-A, fluorescein isothiocyanate-A; $\mathrm{PI}-\mathrm{A}$, propidium iodide-A; LO2, human normal liver cell.

iodide staining (Figure 2B). We next determined whether Z-VAD-FMK suppressed theaflavin-induced activation of caspase-3 using Western blotting. Theaflavin-induced activation of caspase- 3 was partially reversed by pretreatment with Z-VAD-FMK (Figure 2C). These data showed that theaflavins induce apoptosis in HCC cells, partially in a caspase-dependent manner in vitro.

\section{Theaflavins inhibit the migration and} invasion abilities of $\mathrm{HCC}$ cells in vitro

We next detected the effect of theaflavins on cell migration using a wound-healing assay, which measured the rate of cell migration into the area of injury created by a sharp object. A slower closure of the scratched "wound" was observed in theaflavin-treated group compared with the control group (Figure 3A and B). 
A

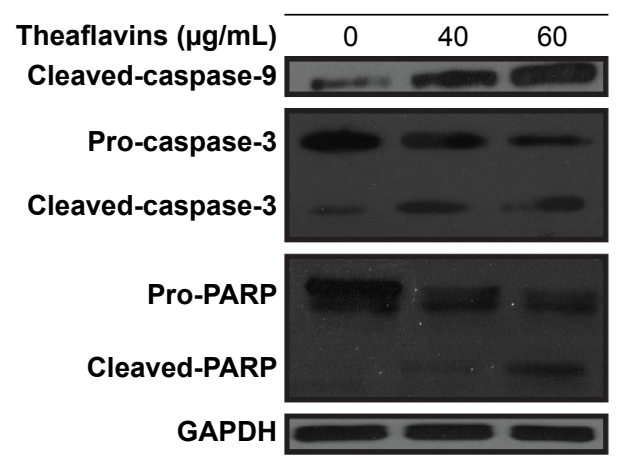

Huh-7

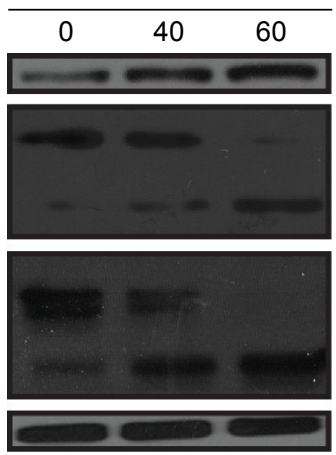

B

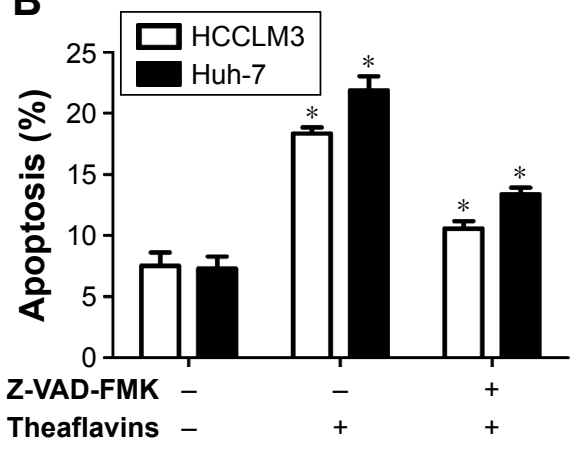

C

HCCLM3

Huh-7

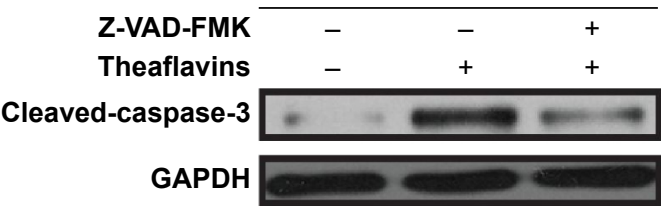

\begin{tabular}{ccc} 
& Huh-7 & \\
\hline- & - & + \\
- & + & + \\
\hline & & \\
\hline & & \\
\hline
\end{tabular}

Figure 2 Theaflavins induce cell apoptosis in a caspase-dependent manner.

Notes: (A) Lysates from HCC cells treated with 0,40 , or $60 \mu \mathrm{g} / \mathrm{mL}$ of theaflavins for 48 hours were probed for cleaved-caspase-9, pro-caspase-3, cleaved-caspase-3, pro-PARP, and cleaved-PARP by Western blotting. (B) Changes in histograms of the apoptotic rate from HCC cells treated with PBS, theaflavins, and theaflavins in combination with the pan-caspase inhibitor (Z-VAD-FMK). (C) Changes in the expression of cleaved-caspase- 3 from HCC cells treated with PBS, theaflavins, and theaflavins in combination with pan-caspase inhibitor (Z-VAD-FMK). Data are expressed as the mean \pm SD of three independent experiments. $* P<0.05$.

Abbreviations: HCC, hepatocellular carcinoma; PBS, phosphate-buffered saline; SD, standard deviation; PARP, poly (ADP-ribose) polymerase; GAPDH, glyceraldehyde 3-phosphate dehydrogenase.

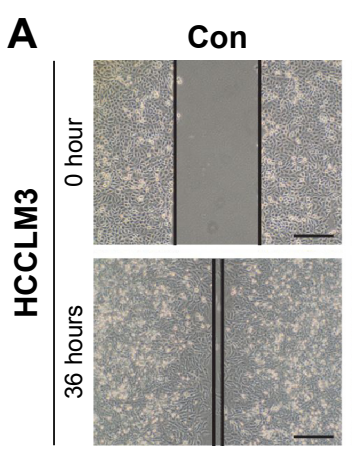

B

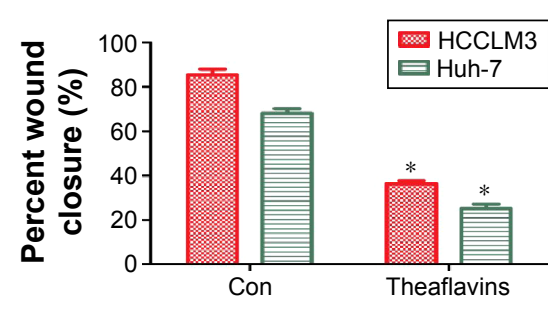

D

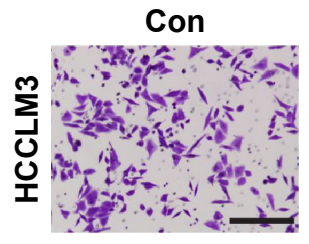

Theaflavins

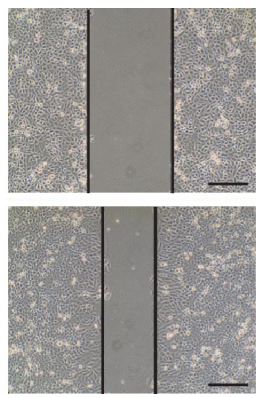

Theaflavins

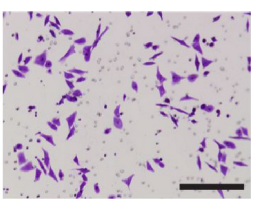

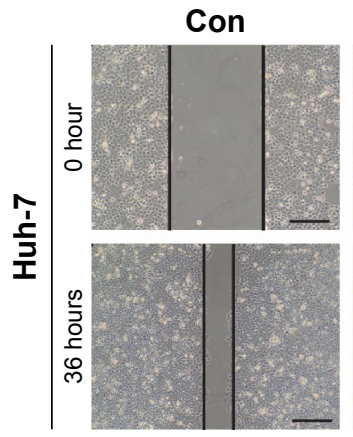

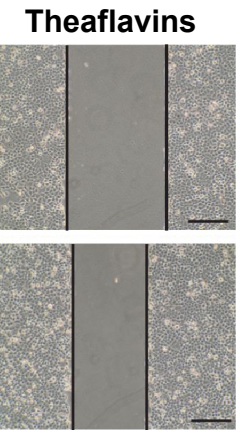

C
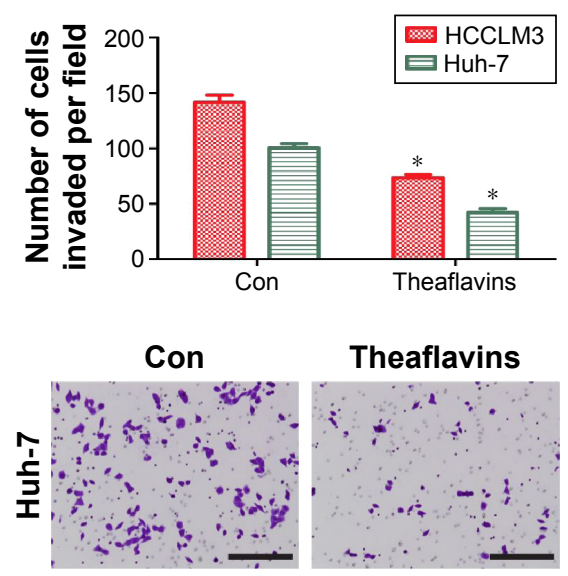

Figure 3 Theaflavins inhibit the migration and invasion abilities of HCC cells in vitro.

Notes: (A) Representative images of cell migration using a wound-healing assay after treatment with $20 \mu \mathrm{g} / \mathrm{mL}$ of theaflavins. Scale bar $=50 \mu \mathrm{m}$. (B) The wound closure was quantified at 36 hours post-wounding by measuring the migrated area. Imagej was used to quantify the wound closure. (C) The number of invaded cells was counted after pretreatment with theaflavins for 24 hours. (D) Representative images of the invasion assay for HCC cells after pretreatment with theaflavins for 24 hours. Scale bar $=100 \mu \mathrm{m}$. Data are expressed as the mean \pm SD of three independent experiments. $* P<0.05$.

Abbreviations: HCC, hepatocellular carcinoma; SD, standard deviation; con, control. 
A Matrigel ${ }^{\circledR}$-coated Transwell ${ }^{\circledR}$ assay was then used to investigate the effect of theaflavins on the invasive abilities of HCC cells. The invasive abilities of HCCLM3 and Huh-7 cells were significantly suppressed after treatment with theaflavins (Figure 3C and D). Similar results were observed in HepG2 cells (Figure S1B).

\section{Theaflavins inhibit constitutive and inducible STAT3 phosphorylation and downregulate the expression of apoptosis- and invasion-related proteins in $\mathrm{HCC}$ cells}

To further evaluate the effect of theaflavins on the apoptosis and invasion of HCC cells, several signal pathways related to the growth and metastasis of HCC were investigated by detecting the expression of STAT3, p-STAT3, AKT, p-AKT, ERK, and p-ERK. Theaflavins showed no effect on the expressions of ERK and p-ERK (data not shown) and a slight effect on the expression of p-AKT (Figure 4A). However, the expression of p-STAT3 was apparently decreased after treatment with theaflavins (Figure 4A). Theaflavins also downregulated the STAT3-regulated gene products, including antiapoptotic proteins (Bcl-2 and Survivin) and invasion-related proteins (MMP-2 and MMP-9) (Figure 4A). We then determined whether theaflavins could suppress IL-6-induced STAT3 phosphorylation in HCC cells. HCC cells were pretreated with theaflavins for 24 hours and then stimulated with IL-6 $(10 \mathrm{ng} / \mathrm{mL})$ for 2 hours. IL-6-induced STAT3 phosphorylation was partially reversed by theaflavins (Figure 4B). To further confirm the effects of theaflavins on apoptosis and invasion of HCC cells, HCCLM3 cells were treated with theaflavins and a STAT3-specific inhibitor (NSC 74859). NSC 74859 enhanced the effects of theaflavins on apoptosis and invasion of HCC cells (Figure 4C and D). Together, these results suggest that the STAT3 pathway is likely an important target of theaflavins in HCC cells.

\section{Theaflavins inhibit tumor growth and metastasis of $\mathrm{HCC}$ in vivo}

We established an orthotopic model using HCCLM3 cells to determine the effects of theaflavins on HCC growth in vivo. Compared with the control group, treatment with theaflavins resulted in a significant decrease in tumor size (Figure 5A). We further evaluated the effect of theaflavins on the expression of Ki-67 (a marker of proliferation) in HCC tumor tissues by immunohistochemical analyses and found that theaflavins significantly decreased the number of Ki-67-positive tumor cells, compared with the control group (Figure 5B). We then performed terminal deoxynucleotidyl transferase dUTP nick end-labeling assays to detect the effect of theaflavins on apoptosis in vivo and found that theaflavins significantly increased the number of apoptotic cells in tumor tissues, compared with the control group (Figure 5C). The effects of theaflavins on the metastatic ability of $\mathrm{HCC}$ were determined in vivo by injecting HCCLM3 cells into the tail vein of nude mice to establish a lung metastasis model. Necropsy after 6 weeks revealed that the theaflavin-treated group had less and smaller lung metastases, compared with the control group (Figure 5D). Furthermore, we detected the expression of tumor-related proteins in nude mice by using Western blotting, and the results were consistent with in vitro data (Figure 5E). In summary, these results showed the ability of theaflavins to suppress tumor growth and metastasis of HCC in vivo.

\section{Discussion}

The main goal of this study was to investigate whether theaflavins exert antitumor effects on HCC. We showed that theaflavins inhibited proliferation, migration, and invasion, and induced apoptosis in HCC in vitro and in vivo. The underlying mechanisms may be, at least in part, due to the inhibition of STAT3 activation and downregulation of its gene products.

Black tea is one of the most popular beverages in the world and has been reported to possess antitumor activity in various cancers. ${ }^{17}$ Theaflavins, the major black tea polyphenols, are thought to exhibit antitumor effects due to their ability to suppress proliferation and/or induce cell cycle arrest and apoptosis through diverse mechanisms. In human prostate carcinoma PC-3 cells, theaflavins induced G2/M arrest by modulating expression of $\mathrm{p} 21 \mathrm{waf} 1 / \mathrm{cip} 1, \operatorname{cdc} 25 \mathrm{C}$, and cyclin B. ${ }^{12}$ Additionally, a recent study reported that theaflavins target Fas/caspase- 8 and Akt/Bad pathways to induce apoptosis in p53-mutated human breast cancer cells. ${ }^{10}$ In our study, theaflavins inhibited proliferation and promoted apoptosis in HCC in vitro but showed no apparent effect on the cell cycle. Furthermore, theaflavins did not decrease the viability of the normal liver cell line, LO2, suggesting that they are compounds that specifically inhibit the proliferation of HCC with negligible side effects. Biochemically, theaflavins increase the expression of cleaved-caspase-3, cleaved-caspase-9, and cleaved-PARP, which are critical factors in the caspase pathway. More importantly, a pancaspase inhibitor (Z-VAD-FMK) can reverse the effects of theaflavins on apoptosis and the expression of cleavedcaspase- 3 in HCC cells.

The IL-6/STAT3 pathway plays an important role in human cancers. ${ }^{18}$ STAT3 can be activated by the IL- 6 cytokine 
A

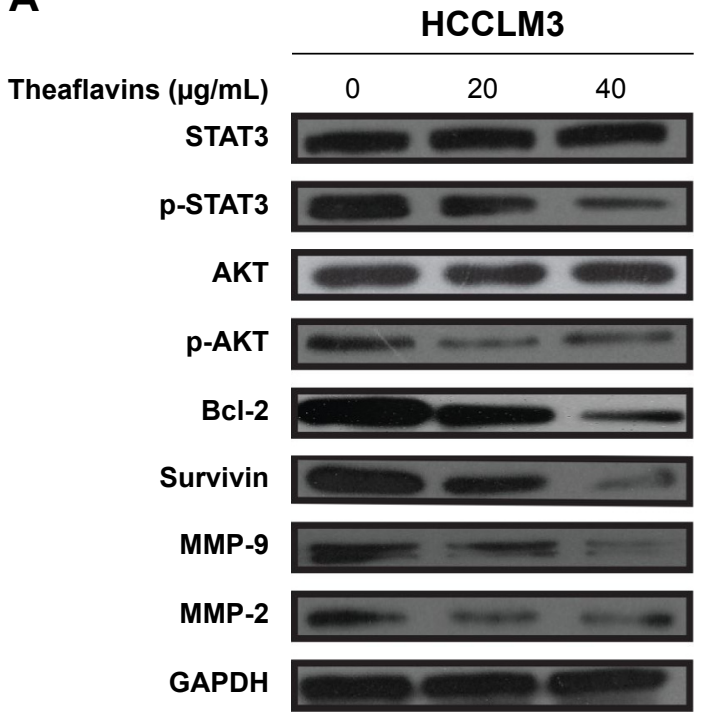

B

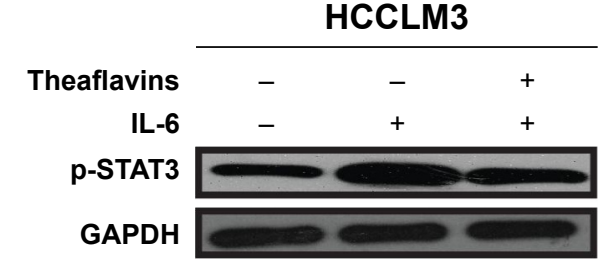

D

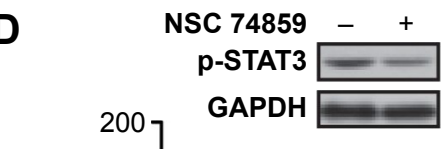

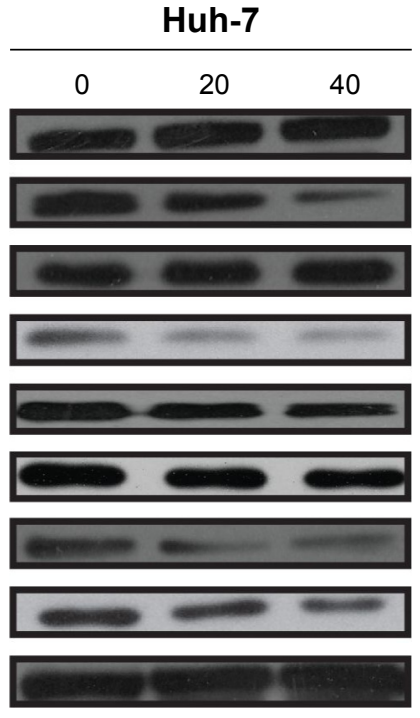

Huh-7
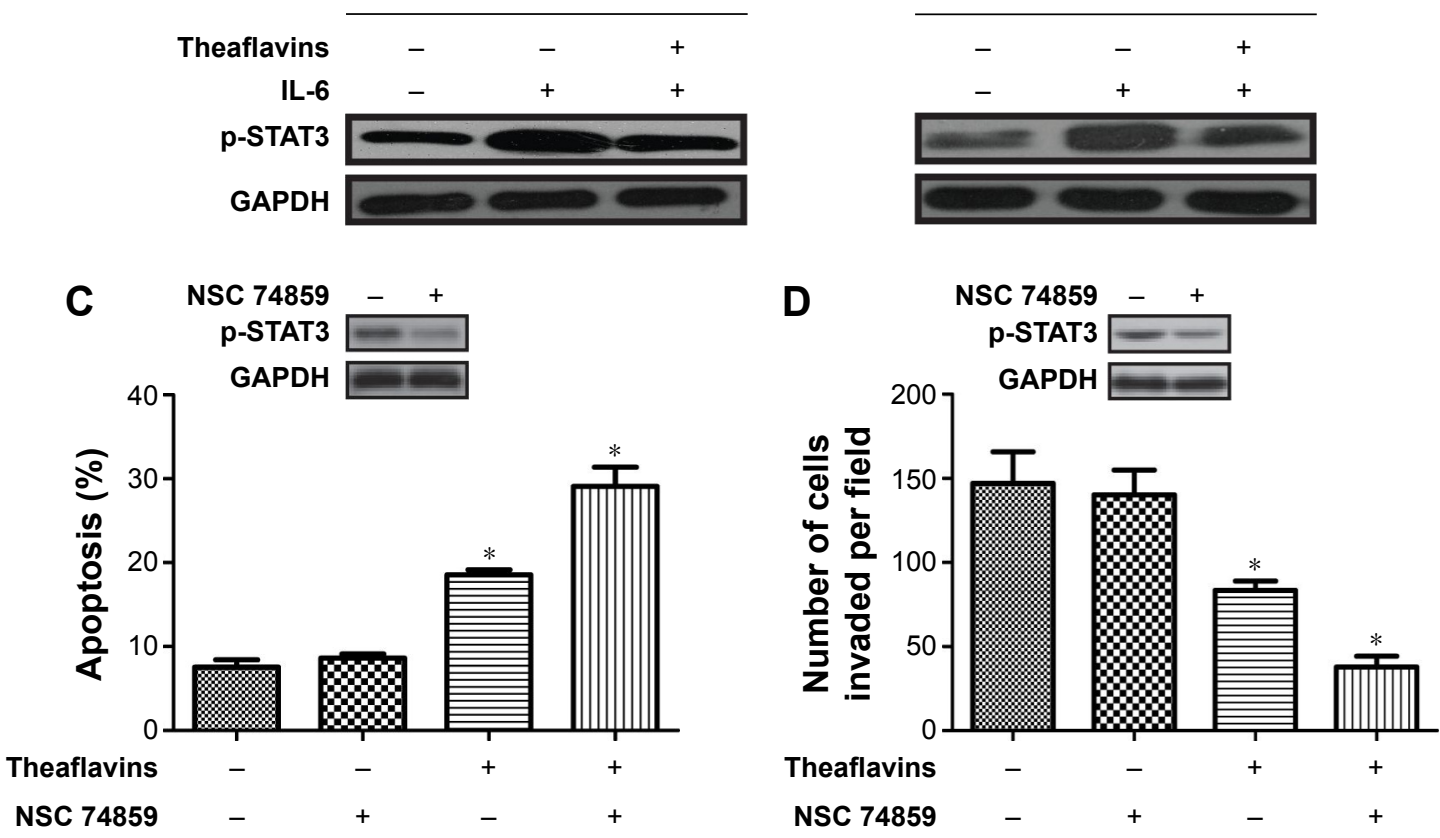

Figure 4 Theaflavins inhibit constitutive and inducible STAT3 phosphorylation, and downregulate the expression of antiapoptotic and invasion-related proteins in HCC cells.

Notes: (A) Lysates from HCC cells treated with 0,20 , or $40 \mu \mathrm{g} / \mathrm{mL}$ of theaflavins for 48 hours were probed for the antiapoptotic proteins by Western blotting. (B) Theaflavins reduced IL-6-induced STAT3 phosphorylation in HCC cells as shown by the Western blotting. (C) HCCLM3 cells were treated with theaflavins (40 $\mu$ g/mL) and NSC $74859(50 \mu \mathrm{M})$ for 48 hours, and cell apoptosis was determined using a flow cytometry assay. (D) HCCLM3 cells were treated with theaflavins (40 $\mu$ g/mL) and NSC $74859(50 \mu \mathrm{M})$ for 48 hours, and the number of invaded cells was determined using an invasion assay. Data are expressed as the mean \pm SD of three independent experiments. $* P<0.05$.

Abbreviations: HCC, hepatocellular carcinoma; SD, standard deviation; STAT3, signal transducer and activator of transcription 3; GAPDH, glyceraldehyde 3-phosphate dehydrogenase; IL, interleukin.

and leptin as well as other growth factors, including epidermal growth factor receptor and platelet-derived growth factor receptor through tyrosine phosphorylation. ${ }^{19}$ After dimerization, STAT3 translocates into the nucleus where it activates the transcription of its target genes by binding to promoter sequences. ${ }^{20,21}$ Many target genes of STAT3 play critical roles in the growth and metastasis of various tumors. ${ }^{22-24} \mathrm{In}$ addition, accumulating data suggest that aberrant expression of STAT3 signaling results in the progression of $\mathrm{HCC}$, with STAT3 inhibitors having antitumor effects on HCC..$^{25-30}$ We therefore propose that STAT3 can be a therapeutic target for HCC treatment. When we tried to find the specific mechanism by which theaflavins inhibit the growth and metastasis of HCC, several signal pathways related to the growth and 
A
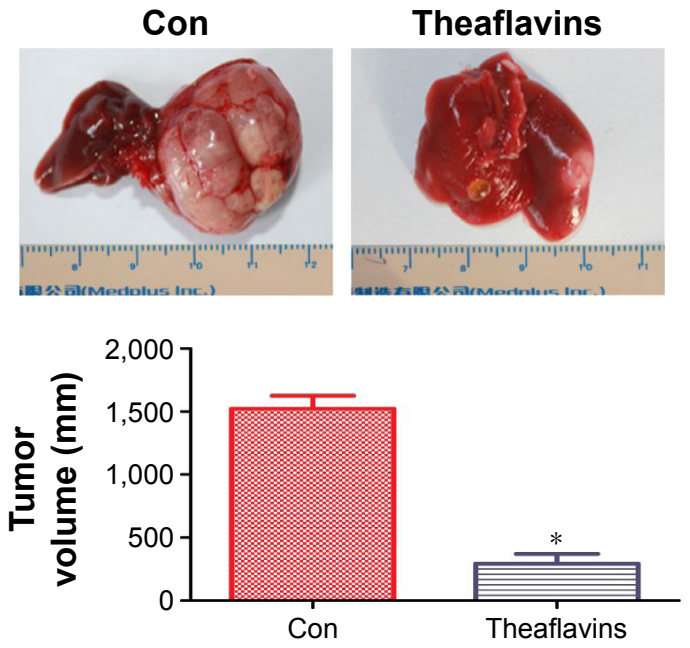

C
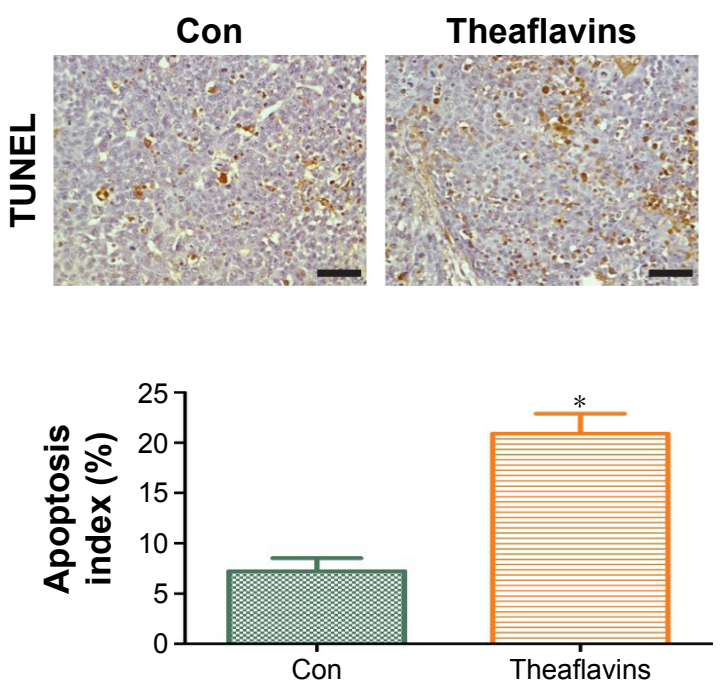

E

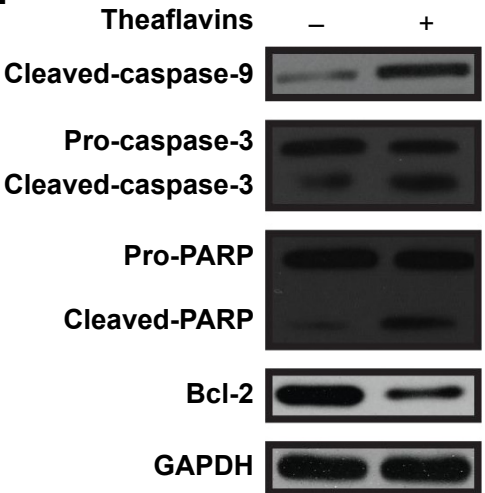

B
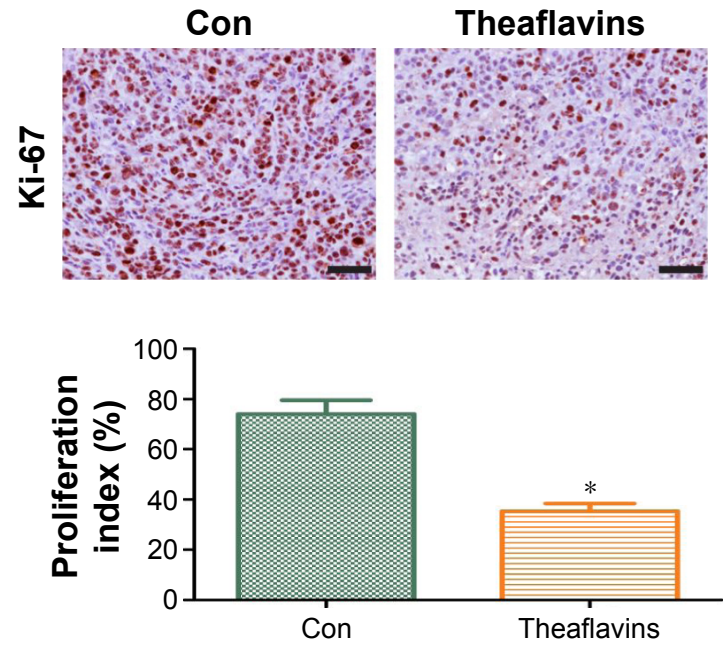

D
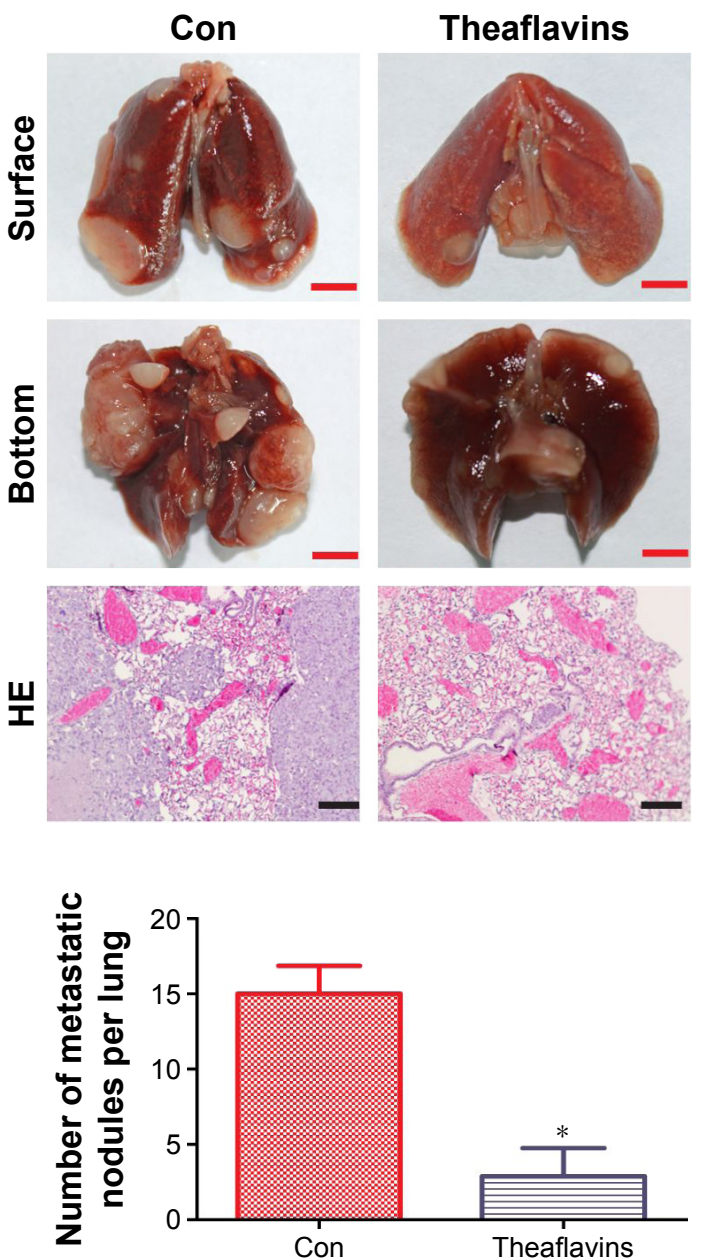

Figure 5 Theaflavins inhibit tumor growth and metastasis of $\mathrm{HCC}$ in vivo.

Notes: (A) Representative images of gross specimens. Tumor volumes were detected in each group ( $\mathrm{n}=6$ ). (B) Immunohistochemical analysis of Ki-67 for cell proliferation. Ki-67-positive cells were quantitated. Scale bar $=100 \mu \mathrm{m}$. (C) TUNEL analysis of apoptotic cells. Results are expressed as the apoptosis index. Scale bar $=100 \mu \mathrm{m}$. (D) Representative images of gross specimens and hematoxylin and eosin staining of lung tissue samples. The number of metastatic lung foci was detected in each group. Black scale bar $=50 \mu \mathrm{m}$. Red scale bar $=0.5 \mathrm{~cm}$. (E) Indicated proteins were detected using Western blotting of tumor samples. Data are expressed as the mean \pm SD of three independent experiments. $* P<0.05$.

Abbreviations: HCC, hepatocellular carcinoma; TUNEL, terminal deoxynucleotidyl transferase dUTP nick end labeling; SD, standard deviation; con, control; HE, hematoxylin and eosin; PARP, poly (ADP-ribose) polymerase; GAPDH, glyceraldehyde 3-phosphate dehydrogenase; STAT3, signal transducer and activator of transcription 3. 
metastasis of HCC were investigated by detecting the expressions of STAT3, p-STAT3, AKT, p-AKT, ERK, and p-ERK. Theaflavins reduced the expression of $\mathrm{p}$-AKT but to a less extent. Theaflavins showed no effect on the expressions of ERK and p-ERK. Previous studies have reported that theaflavins exhibit antitumor activity by inhibiting the phosphatidylinositol-3-kinase/protein kinase B and ERK signal pathways. ${ }^{10,31}$ The reason for the contradictory conclusions may be that theaflavins exhibit antitumor activity in a contextdependent manner. Notably, the expression of p-STAT3 was significantly decreased after theaflavins treatment. Concurrently, theaflavins reduced the expression of downstream proteins regulated by STAT3, such as Bcl-2, Survivin, MMP-2, and MMP-9. We therefore conclude that the inhibitory effects of theaflavins on HCC can mainly be attributed to theaflavininduced blockage of the STAT3 pathway.

Previous studies reported the antitumor activity of theaflavins using in vitro experiments. ${ }^{10-12}$ In this study, an orthotopic model and a lung metastasis model of HCC were established in addition to an in vitro cell model. The results showed that theaflavins inhibited proliferation and induced apoptosis in tumor tissues (associated with less Ki-67 immunostaining and a larger number of apoptotic cells). Additionally, the theaflavin-treated group showed less and smaller lung metastases, compared with the control group. Similar to the in vitro study, theaflavins could also inhibit the activation of the STAT3 pathway in vivo. These in vivo data further support the hypothesis that the antitumor effects of theaflavins on HCC are mediated by the suppression of STAT3 activation.

\section{Conclusion}

We have shown that theaflavins potently inhibit constitutive and IL-6-inducible STAT3 activation, which may lead to the suppression of growth and metastasis of HCC. Although the role of theaflavins in HCC still needs to be demonstrated in clinical trials, we propose that theaflavins may be a potential agent for prevention and therapy of HCC.

\section{Disclosure}

The authors report no conflicts of interest in this work.

\section{References}

1. Singal AG, El-Serag HB. Hepatocellular carcinoma from epidemiology to prevention: translating knowledge into practice. Clin Gastroenterol Hepatol. 2015;13(12):2140-2151.

2. Lee JS, Thorgeirsson SS. Genome-scale profiling of gene expression in hepatocellular carcinoma: classification, survival prediction, and identification of therapeutic targets. Gastroenterology. 2004;127(5 Suppl 1): S51-S55.
3. Thorgeirsson SS, Lee JS, Grisham JW. Molecular prognostication of liver cancer: end of the beginning. J Hepatol. 2006;44(4):798-805.

4. Cervello M, McCubrey JA, Cusimano A, Lampiasi N, Azzolina A, Montalto G. Targeted therapy for hepatocellular carcinoma: novel agents on the horizon. Oncotarget. 2012;3(3):236-260.

5. Clardy J, Walsh C. Lessons from natural molecules. Nature. 2004; 432(7019):829-837.

6. Tanaka T, Inoue K, Betsumiya Y, Mine C, Kouno I. Two types of oxidative dimerization of the black tea polyphenol theaflavin. J Agric Food Chem. 2001;49(12):5785-5789.

7. Leung LK, Su Y, Chen R, Zhang Z, Huang Y, Chen ZY. Theaflavins in black tea and catechins in green tea are equally effective antioxidants. J Nutr. 2001;131(9):2248-2251.

8. Hasaniya N, Youn K, Xu M, Hernaez J, Dashwood R. Inhibitory activity of green and black tea in a free radical-generating system using 2-amino-3-methylimidazo[4,5-f]quinoline as substrate. Jpn J Cancer Res. 1997;88(6):553-558.

9. Shiraki M, Hara Y, Osawa T, Kumon H, Nakayama T, Kawakishi S Antioxidative and antimutagenic effects of theaflavins from black tea. Mutat Res. 1994;323(1-2):29-34.

10. Lahiry L, Saha B, Chakraborty J, et al. Theaflavins target Fas/caspase-8 and $\mathrm{Akt} / \mathrm{pBad}$ pathways to induce apoptosis in p53-mutated human breast cancer cells. Carcinogenesis. 2010;31(2):259-268.

11. Adhikary A, Mohanty S, Lahiry L, Hossain DM, Chakraborty S, Das T. Theaflavins retard human breast cancer cell migration by inhibiting NF-kappaB via p53-ROS cross-talk. FEBS Lett. 2010;584(1):7-14.

12. Prasad S, Kaur J, Roy P, Kalra N, Shukla Y. Theaflavins induce G2/M arrest by modulating expression of $\mathrm{p} 21 \mathrm{waf} 1 / \mathrm{cip} 1, \mathrm{cdc} 25 \mathrm{C}$ and cyclin B in human prostate carcinoma PC-3 cells. Life Sci. 2007;81(17-18): 1323-1331.

13. Sun S, Pan S, Miao A, et al. Active extracts of black tea (Camellia Sinensis) induce apoptosis of PC-3 prostate cancer cells via mitochondrial dysfunction. Oncol Rep. 2013;30(2):763-772.

14. Sil H, Sen T, Moulik S, Chatterjee A. Black tea polyphenol (theaflavin) downregulates MMP-2 in human melanoma cell line A375 by involving multiple regulatory molecules. J Environ Pathol Toxicol Oncol. 2010; 29(1):55-68.

15. Lahiry L, Saha B, Chakraborty J, et al. Contribution of p53-mediated Bax transactivation in theaflavin-induced mammary epithelial carcinoma cell apoptosis. Apoptosis. 2008;13(6):771-781.

16. Weerawatanakorn M, Lee YL, Tsai CY, et al. Protective effect of theaflavin-enriched black tea extracts against dimethylnitrosamineinduced liver fibrosis in rats. Food Funct. 2015;6(6):1832-1840.

17. Yang CS, Wang ZY. Tea and cancer. J Natl Cancer Inst. 1993;85(13): 1038-1049.

18. Chai EZ, Shanmugam MK, Arfuso F, et al. Targeting transcription factor STAT3 for cancer prevention and therapy. Pharmacol Ther. 2016;162:86-97.

19. Schuringa JJ, Wierenga AT, Kruijer W, Vellenga E. Constitutive Stat3, Tyr705, and Ser727 phosphorylation in acute myeloid leukemia cells caused by the autocrine secretion of interleukin-6. Blood. 2000; 95(12):3765-3770.

20. Zhong Z, Wen Z, Darnell JE Jr. Stat3: a STAT family member activated by tyrosine phosphorylation in response to epidermal growth factor and interleukin-6. Science. 1994;264(5155):95-98.

21. Hemmann U, Gerhartz C, Heesel B, et al. Differential activation of acute phase response factor/Stat3 and Stat1 via the cytoplasmic domain of the interleukin 6 signal transducer gp130. II. Src homology SH2 domains define the specificity of stat factor activation. J Biol Chem. 1996; 271(22):12999-13007.

22. Lai PS, Rosa DA, Magdy Ali A, et al. A STAT inhibitor patent review: progress since 2011. Expert Opin Ther Pat. 2015;25(12):1397-1421.

23. Peyser ND, Grandis JR. Critical analysis of the potential for targeting STAT3 in human malignancy. Onco Targets Ther. 2013;6:999-1010.

24. Shodeinde AL, Barton BE. Potential use of STAT3 inhibitors in targeted prostate cancer therapy: future prospects. Onco Targets Ther. 2012;5: $119-125$. 
25. Zuo M, Li C, Lin J, Javle M. LLL12, a novel small inhibitor targeting STAT3 for hepatocellular carcinoma therapy. Oncotarget. 2015;6(13): 10940-10949.

26. Subramaniam A, Shanmugam MK, Ong TH, et al. Emodin inhibits growth and induces apoptosis in an orthotopic hepatocellular carcinoma model by blocking activation of STAT3. Br J Pharmacol. 2013; 170(4):807-821.

27. Rajendran P, Li F, Manu KA, et al. $\gamma$-Tocotrienol is a novel inhibitor of constitutive and inducible STAT3 signalling pathway in human hepatocellular carcinoma: potential role as an antiproliferative, proapoptotic and chemosensitizing agent. Br J Pharmacol. 2011;163(2): 283-298.

28. Liu Y, Liu A, Xu Z, et al. XZH-5 inhibits STAT3 phosphorylation and causes apoptosis in human hepatocellular carcinoma cells. Apoptosis. 2011;16(5):502-510.
29. Yoshida T, Hanada T, Tokuhisa T, et al. Activation of STAT3 by the hepatitis $\mathrm{C}$ virus core protein leads to cellular transformation. J Exp Med. 2002;196(5):641-653.

30. Subramaniam A, Shanmugam MK, Perumal E, et al. Potential role of signal transducer and activator of transcription (STAT)3 signaling pathway in inflammation, survival, proliferation and invasion of hepatocellular carcinoma. Biochim Biophys Acta. 2013;1835(1):46-60.

31. Bhattacharya U, Halder B, Mukhopadhyay S, Giri AK. Role of oxidationtriggered activation of JNK and p38 MAPK in black tea polyphenols induced apoptotic death of A375 cells. Cancer Sci. 2009;100(10): 1971-1978 


\section{Supplementary material}

A

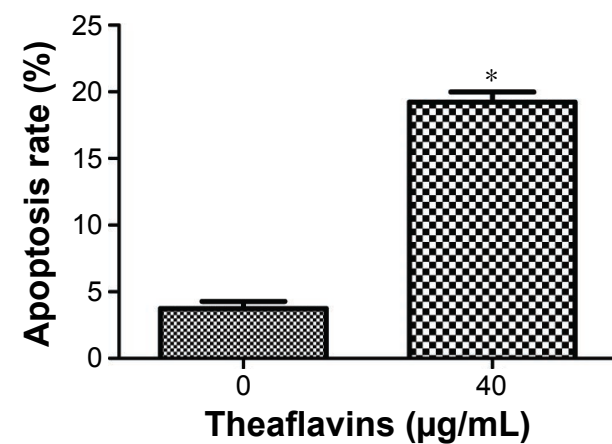

B

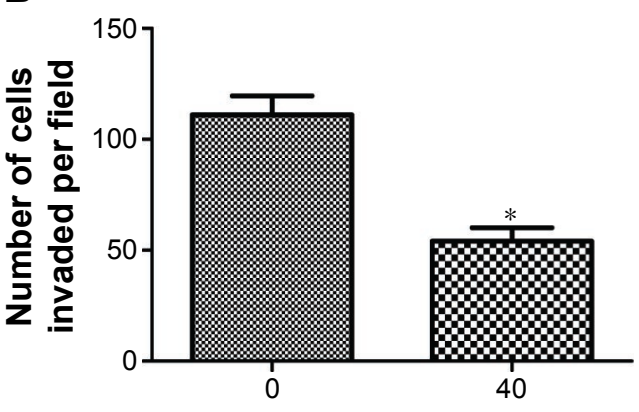

Theaflavins $(\mu \mathrm{g} / \mathrm{mL})$

Figure SI Theaflavins promote apoptosis and inhibit the invasion abilities of HepG2 cells in vitro.

Notes: (A) Flow cytometry results of annexin V-Pl-stained HepG2 cells after exposure to $40 \mu \mathrm{g} / \mathrm{mL}$ of theaflavins for 48 hours. (B) The number of invaded cells was counted after pretreatment with theaflavins for 24 hours. Data are expressed as the mean \pm SD of three independent experiments. $* P<0.05$.

Abbreviations: SD, standard deviation; PI, propidium iodide.

\section{Publish your work in this journal}

OncoTargets and Therapy is an international, peer-reviewed, open access journal focusing on the pathological basis of all cancers, potential targets for therapy and treatment protocols employed to improve the management of cancer patients. The journal also focuses on the impact of management programs and new therapeutic agents and protocols on
Dovepress

patient perspectives such as quality of life, adherence and satisfaction. The manuscript management system is completely online and includes a very quick and fair peer-review system, which is all easy to use. Visit http://www.dovepress.com/testimonials.php to read real quotes from published authors. 